The number of taxa unknown to science is particularly more for underground biota, especially nematodes, with only an estimated
ISSN

Online 0974-7907 Print 0974-7893 $3 \%$ of the world's species having been studied and described (Barker et al. 1994). Nematodes are biologically diverse and versatile, occupying diverse habitats and constitute nearly $90 \%$ of all metazoans in number (Hugot et al. 2001). Soil inhabiting nematodes predominate over all other soil animals, both in number and species. Nematodes possess several attributes that make them useful ecological indicators (Bongers 1990; Freckman 1988; Neher 2001). They are very important and beneficial in the decomposition of organic matter and recycling of soil nutrients in terrestrial ecosystem. A review of the literature by Boag \& Yeates (1998) regarding nematode diversity, stressed the critical lack of information in tropical areas.

Goa is the smallest agrarian state of India by area, but has rich flora and fauna, owing to its location on the Western Ghats which has been internationally recognized as a Biodiversity Hotspot. Extensive work has been done on the fauna of Goa (Director ZSI 2008). However, groups such as nematodes are practically ignored and unrecorded in all biodiversity studies. Owing to their microscopic size and hidden life they might have been totally neglected. There are about 28,000 species of nematodes that have been recorded globally and the estimated diversity is to be about 1,000,000 (Hugot et al. 2001). On record, so far, only 10 species of nematodes have been recorded from Goa (goafoundation.org). To fill in the lacunae in the knowledge about the diversity

\section{A PRELIMINARY SURVEY ON SOIL AND PLANT PARASITIC NEMATODES OF SOUTHERN GOA, INDIA}

\section{A.C. Maria Lizanne ${ }^{1} \&$ Irvathur Krishnananda Pai ${ }^{2}$}

${ }^{1}$ Carmel College of Arts, Science \& Commerce for Women, Nuvem, Salcette, Goa 403604, India ${ }^{2}$ Department of Zoology, Goa University, Taleigao Plateau, Goa 403206, India ${ }^{1}$ marializanne@gmail.com (corresponding author), ${ }^{2}$ ikpai@unigoa.ac.in

of nematodes in Goa, the present study was carried out to explore the nematode diversity of the state. Based on the results obtained, the present paper reports the diversity of soil inhabiting nematode fauna from South Goa District of Goa State (Fig. 1).

Materials and Methods: Soil samples were collected from August 2011 to November 2011 and from July 2012 to December 2012 from five talukas-Canacona, Marmagoa, Quepem, Salcette and Sanguem. About 50 soil samples were randomly collected from five different villages of each taluka covering 20 landscape elements (Table 1). From each type of landscape, soil samples of about 500-1000 g near the roots of the plants were collected by taking care to avoid the top soil of about 10-15 cm depth. Each sample was collected in a selfsealing plastic bag with a label containing necessary field information. They were either processed immediately or stored in the refrigerator at $4^{\circ} \mathrm{C}$ and processed later. The processing involved soaking the samples in freshwater for a few minutes and then collecting the nematodes from these samples by Cobb's decanting and sieving method (Cobb 1919), followed by the modified Baermann's funnel method (Thorne 1961). The nematodes that were isolated were fixed in warm $4 \%$ formalin and processed by slow glycerine method

DOI: http://dx.doi.org/10.11609/JoTT.o3568.5400-12 | ZooBank: urn:Isid:zoobank.org:pub:FEF74B33-6C2F-487A-8D89-1F86FBA25100

Editor: N. Mohilal Meitei, Manipur University, Canchipur, India.

Date of publication: 26 January 2014 (online \& print)

Manuscript details: Ms \# 03568 | Received 26 March 2013 | Final received 29 July 2013 | Finally accepted 05 November 2013

Citation: Lizanne, A.C.M. \& I.K. Pai (2014). A preliminary survey on soil and plant parasitic nematodes of southern Goa, India. Journal of Threatened Taxa 5(17): 5400-5412; http://dx.doi.org/10.11609/JoTT.03568.5400-12

Copyright: (c) Lizanne \& Pai 2014. Creative Commons Attribution 3.0 Unported License. JoTT allows unrestricted use of this article in any medium, reproduction and distribution by providing adequate credit to the authors and the source of publication.

Funding: The Apostolic Carmel Educational Society of Goa.

Competing Interest: The authors declare no competing interests.

Acknowledgements: The authors wish to express their sincere gratitude to Prof. M.S. Jairajpuri, Prof. Irfan Ahmad and the research scholars of the Department of Zoology, Aligarh Muslim University, Aligarh, for their expertise. The authors also extend their thanks to Dr. Divakar Mesta, Carmel College for Women for his valuable inputs and all those who helped in the collection of the samples. 


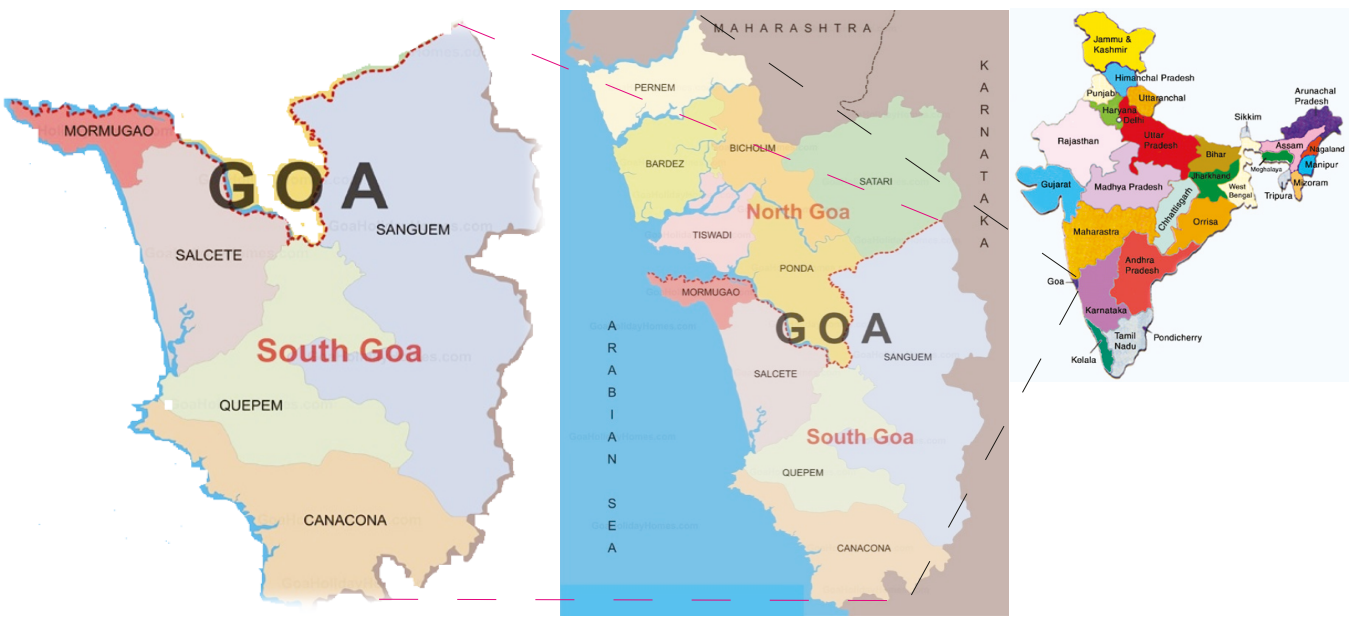

Figure 1. Maps of India, Goa and South Goa District with the talukas

Table 1. Details of sampling sites and various landscapes

\begin{tabular}{|c|c|c|}
\hline & Talukas: Villages & Landscapes \\
\hline \multirow[t]{5}{*}{1} & $\begin{array}{l}\text { Marmagoa: } \\
\text { i) Chicalim }\end{array}$ & Flower gardens, Banana grove \\
\hline & ii) Consua & Bushy plants, Radish plantation \\
\hline & iii) Sao Jacinto Island & Coconut plantation, \\
\hline & iv) Cortalim & Cashew plantation, Banana plantation \\
\hline & v) Vasco & Coconut plantation, Paddy fields \\
\hline \multirow[t]{5}{*}{2} & $\begin{array}{l}\text { Salcette: } \\
\text { i) Raia }\end{array}$ & Flower gardens, Arecanut plantation \\
\hline & ii) Nuvem & $\begin{array}{l}\text { Banana plantation, Cashew plantation, } \\
\text { Acacia plantation }\end{array}$ \\
\hline & iii) Carmona & $\begin{array}{l}\text { Casuarina plantation, near roots of } \\
\text { vegetables plants - chillies, tomatoes, } \\
\text { brinjal, etc. }\end{array}$ \\
\hline & iv) Curtorim & Paddy fields, roadside weeds \\
\hline & v) Loutolim & $\begin{array}{l}\text { Rubber plantation, Chikoo (sapota) } \\
\text { plantation }\end{array}$ \\
\hline \multirow[t]{5}{*}{3} & $\begin{array}{l}\text { Quepem: } \\
\text { i) Ambaulim }\end{array}$ & Bamboo reeds, Terminalia spp. \\
\hline & ii) Balli & Scrub jungle, roadside weeds \\
\hline & iii) Quepem & Teak plantation, Acacia plantation \\
\hline & iv) Avedem & Paddy fields, Cashew plantation \\
\hline & v) Xeldem & Mango plantation, Jackfruit plantation \\
\hline \multirow[t]{5}{*}{4} & $\begin{array}{l}\text { Canacona: } \\
\text { i) Agonda }\end{array}$ & $\begin{array}{l}\text { Forest area, Bamboo reeds, Cashew } \\
\text { plantation }\end{array}$ \\
\hline & ii) Loliem & Arecanut plantation, Banana plantation \\
\hline & iii) Cabo da Rama & Casuarina plantation, Bushy plants \\
\hline & iv) Butpal & Vegetable plantation \\
\hline & v) Palolem & Casuarina plantation \\
\hline \multirow[t]{5}{*}{5} & $\begin{array}{l}\text { Sanguem: } \\
\text { i) Darbandora }\end{array}$ & Coconut plantation \\
\hline & ii) Molem & Forest area \\
\hline & iii) Sanvordem & Acacia plantation \\
\hline & iv) Colem & Forest area \\
\hline & v) Rivona & Acacia plantation, coconut plantation \\
\hline
\end{tabular}

(Seinhorst 1959). They were mounted in dehydrated glycerine after $4-5$ weeks of dehydration and permanent slides of the specimens were prepared using paraffin wax ring method and numbered serially (de Maeseneer \& d'Herde 1963). For classification the nematodes were listed according to Goodey (1963), Jairajpuri \& Khan (1982), Jairajpuri \& Ahmad (1992), Andrassy (1999), Siddiqi (2000), Choudhary et al. (2010) and websites of NEMAPLEX.

Results: In the present study, about 400 permanent slides were prepared. A total of 52 species of nematodes belonging to seven orders were recorded, of which 27 species were from order Dorylaimida, six from Mononchida, nine from Tylenchida, three from Alaimida, four from Rhabditida, two from Enoplida, and one from Araeolaimida. The detailed information of these 52 species is as follows:

\section{Phylum Nematoda (Rudolphi, 1808) Lankester, 1877 I Order Dorylaimida Pearse, 1942}

Suborder Dorylaimina Pearse, 1936

Superfamiliy Dorylaimoidea De Man, 1876

Family Dorylaimidae De Man, 1876

Subfamily Laimydorinae Andrassy, 1969

Genus Amphidorylaimus Andrassy, 1960

1. Amphidorylaimus infecundus (Cobb, 1936) Andrassy, 1960

Genus Afrodorylaimus Andrassy, 1960

2. Afrodorylaimus bwana Andrassy, 1964

Genus Prodorylaimus Andrassy, 1959

3. Prodorylaimus longicaudatus (Butschli, 1874) 
Andrassy, 1959

4. Prodorylaimus obesus Ahmad \& Jairajpuri, 1982

Genus Mesodorylaimus Andrassy, 1959

5. Mesodorylaimus mesonyctius (Kreis, 1930) Andrassy, 1959

Subfamily Thornenematinae Siddiqi, 1969

Genus Thornenema Andrassy, 1959

6. Thornenema baldum (Thorne, 1939) Andrassy, 1959

7. Thornenema lissum (Thorne, 1939) Andrassy, 1959

Genus Coomansinema Ahmad \& Jairajpuri, 1989

8. Coomansinema dimorphicauda Ahmad \& Jairajpuri 1989

Family Qudsianematidae Jairajpuri, 1965

Subfamily Qudsianematinae Jairajpuri, 1965

Genus Baqriella Ahmad \& Jairajpuri, 1988

9. Baqriella qaiseri Ahmad \& Jairajpuri, 1988

Genus Ecumenicus Thorne, 1974

10. Ecumenicus monohystera (De Man, 1880) Thorne, 1974

Genus Labronema Thorne, 1974

11. Labronema ferox Thorne, 1939

Genus Eudorylaimus Andrassy, 1959

12. Eudorylaimus himalus Jairajpuri \& Ahmad, 1982

Subfamily Discolaiminae Siddiqi, 1969

Genus Discolaimus Cobb, 1913

13. Discolaimus texanus Cobb, 1913

14. Discolaimus laksi Khan \& Laha, 1982

Family Nordiidae Jairajpuri \& Siddiqi, 1964

Subfamily Pungentinae Siddiqi, 1969

Genus Enchodelus Thorne, 1939

15. Enchodelus (Parenchodelus) constrictus Jairajpuri \& Loof, 1968

16. Enchodelus (Parenchodelus) longidens Jairajpuri \& Loof, 1968

Subfamily Actinolaimoidinae Jairajpuri \& Ahmad, Genus Oriverutus Siddiqi, 1971

17. Oriverutus labiatus Ahmad \& Jairajpuri, 1987

18. Oriverutus paragus Ahmad \& Jairajpuri, 1987

Family Aporcelaimidae Heyns, 1965

Subfamily Aporcelaiminae Heyns, 1965

Genus Aporcelaimellus Heyns, 1965

19. Aporcelaimellus obscures (Thorne \& Swanger, 1936)
Heyns, 1965

20. Aporcelaimellus baqrii Ahmad \& Jairajpuri, 1982

Genus Aporcelaimus Thorne \& Swanger, 1936

21. Aporcelaimus regius (De Man, 1876) Thorne \& Swanger, 1936

Superfamily Longidoroidea Thorne, 1935

Family Longidoridae thorne, 1935

Subfamily Longidorinae Thorne, 1035

Genus Longidorus Micoletzky, 1922

22. Longidorus brevicaudatus (Schur. Stek, 1951) Khan, 1987

23. Longidorus elongates (De Man, 1876) Thorne \& Swanger, 1936

Family Xiphinematidae Dalmasso, 1969

Subfamily Xiphinematinae Dalmasso, 1969

Genus Xiphinema Cobb, 1913

24. Xiphinema insigne Loos, 1949

25. Xiphinema americanum Cobb, 1913

Superfamily Belondiroidea Thorne, 1939

Family Delondiridae Thorne, 1939

Subfamily Belondirinae Thorne, 1939

Genus Axonchium Cobb, 1920

26. Axonchium (Axonchium) amplicolle Cobb, 1920

27. Axonchium (Epaxonchium) vulvulatum Nair \& Coomans, 1974

\section{Order Mononchida Jairajpuri, 1969}

Suborder Mononchina Kirjanova \& Krall, 1969

Superfamily Mononchoidea Chitwood, 1937

Family Mononchidae Chitwood, 1937

Subfamily Mononchina Chitwood, 1937

Genus Mononchus Bastian, 1865

28. Mononchus aquaticus Coetzee, 1968

29. Mononchus tunbridgensis Bastian, 1865

Family lotonchidae Jairajpuri, 1969

Subfamily lotonchinae Jairajpuri, 1969

Genus lotonchus (Cobb, 1916) Altherr, 1950

30. Iotonchus trichurus (Cobb, 1917) Altherr, 1958

31. Iotonchus indicus Jairajpuri, 1969

Genus Parahadronchus Mulvey, 1978

32. Parahadronchus shakili (Jairajpuri, 1969) Mulvey, 1978

33. Parahadronchus andamanicus (Jairajpuri, 1969) Mulvey, 1978 
III Order Tylenchida Thorne, 1949

Suborder Tylenchina Thorne, 1949

Superfamily Tylenchoidea Orley, 1880

Family Tylenchidae Orley, 1880

Subfamily Tylenchinae Orley, 1880

Genus Tylenchus Bastian, 1865

34. Tylenchus filiformis Butschli, 1873

35. Tylenchus indicus Khan et al., 1969

Genus Ottolenchus Husain \& Khan, 1967

36. Ottolenchus parvus (Siddiqi, 1963) Siddiqi, 1979

Subfamily Boleodorinae Khan, 1964

Genus Psilenchus de Man, 1921

37. Psilenchus minor Siddiqi, 1963

Family Belonolaimidae Whitehead, 1960

Subfamily Telotylenchinae Siddiqi, 1960

Genus Tylenchorhynchus Cobb, 1913

38. Tylenchorhynchus elegans Siddiqi, 1961

Family Hoplolaimidae Filipjev, 1934

Subfamily Hoplolaiminae Filipjev, 1934

Genus Hoplolaimus Daday, 1905

39. Hoplolaimus indicus Sher, 1963

40. Hoplolaimus seinhorsti Luc, 1958

Genus Helicotylenchus Steiner, 1945

41. Helicotylenchus indicus Sddiqi, 1963

Superfamily Criconematoidea Taylor, 1936

Family Criconematidae Taylor, 1936

Subfamily Criconematinae Taylor, 1936

Genus Criconemella De Grisse \& Loof, 1965

42. Criconemella xenoplax (Raski, 1952) Luc \& Raski, 1981

IV Order Alaimida Siddiqi, 1983

Suborder Alaimina Siddiqi, 1983

Family Alaimidae Micoletzky, 1922

Genus Alaimus de Man, 1880

43. Alaimus primitivus de Man, 188

44. Alaimus hamulus Siddiqi \& Husain, 1967

Genus Amphidelus Thorne, 1939

45. Amphidelus novus Baqri \& Jairajpuri, 1968

\section{Order Rhabditida (Orley, 1880) Chitwood, 1933}

Suborder Rhabditina (Orley, 1880) Chitwood, 1933

Superfamily Rhabditoidea (Orley, 1880) Travassos 1920

Family Rhabditidae Orley, 1880
Subfamily Peloderinae Andrassy, 1976

Genus Caenorhabditis (Osche, 1952) Dougherty, 1953

46. Caenorhabditis elegans (Maupas, 1899) Dougherty, 1953

Suborder Cephalobina Filipjev, 1934

Family Cephalobidae Filipjev, 1934

Subfamily Cephalobinae Filipjev, 1934

Genus Cephalobus Bastian, 1865

47. Cephalobus persegnis Bastian, 1865

Subfamily Acrobelinae Thorne, 1937

Genus Acrobeles von Linstow, 1877

48. Acrobeles timmi Chaturvedi \& Khera, 1979

Superfamily Panagrolaimoidea Thorne, 1937

Family Panagrolaimidae Thorne, 1937

Subfamily Panagrolaiminae Thorne, 1937

Genus Panagrolaimus Fuchs, 1930

49. Panagrolaimus fuchsi Ruhm, 1956

VI Order Enoplida (Baird, 1853) Chitwood, 1933

Family Ironidae de Man, 1876

Subfamily Ironinae (De Man, 1876) Micoletzky, 1922

Genus Ironus Bastian, 1865

50. Ironus longicaudatus De Man, 1884

51. Ironus ignavus Bastian, 1865

VII Order Araeolaimida De Coninck and Sch. Stek. 1933 Superfamily Plectoidea (Orley, 1880) Chitwood, 1937

Family Plectidae Orley, 1880

Subfamily Plectinae (Orley, 1880) Micoletzky, 1922

Genus Plectus Bastian, 1865

52. Plectus cirratus Bastian, 1865

\section{Description and Diagnosis \\ I Order Dorylaimida}

Amphidorylaimus infecundus (Cobb, 1936) Andrassy, 1960

Small nematodes less than $1 \mathrm{~mm}$ long, cuticle finely striated, lips well offset by constriction and angular, oesophagus cylindroid with basal expansion up to less than half its length, tail gradually tapering up to anus, similar in sexes

Abbreviations: $\mathrm{L}=$ Total body length; $\mathrm{a}=$ Body length/greatest body width; $\mathrm{b}=$ Body length/neck length; $\mathrm{c}=$ Body length/tail length; $\mathrm{c}^{\prime}=$ Tail length/body width at anus or cloaca; $V=$ Distance of vulva from ant. end $x$ 100/body length; $G^{1}=$ Distance of vulva from ant. ovary x100/body length; $\mathrm{G}^{2}=$ Distance of vulva from post. ovary $\mathrm{x} 100 /$ body length; $A B D=$ Anal Body Diameter 
Locality: Canacona Quepem Salcette Sanguem

Latitude: $15.01415 \mathrm{~N}, \quad 15.2376 \mathrm{~N}, \quad 15.3603 \mathrm{~N}$, $15.1967 \mathrm{~N}$.

Longitude: 74.05711E, 74.2391E, 73.9234E, 74.1195E.

Habitat: Near the roots of vegetable plants, coconut and sapota plantations.

Dimensions: Female: $L=0.91-0.95 \mathrm{~mm}, a=45, b=4.3$, $c=3.5-3.7, V=36-37$, stylet $=10=11 \mu \mathrm{m}$.

\section{Afrodorylaimus bwana Andrassy, 1964}

Body length 2-5 mm, cuticle striated, lip region offset by depression, odontostyle with an aperture about onethird its length, ventromedian supplements closely spaced, tail long and filiform in females and conoid with pointed tip in males.

Locality: Salcette Quepem

Latitude: $15.3603 \mathrm{~N}, 15.2376 \mathrm{~N}$.

Longitude: 73.9234E, 74.2391E.

Habitat: Near the roots of roadside weeds.

Prodorylaimus longicaudatus (Butschli, 1874) Andrassy, 1959

Body $2-5 \mathrm{~mm}$ long, cuticle finely striated, lip region offset by depression, odontostyle with wide lumen odontophore rod-like, female reproductive system amphidelphic, ventromedian supplements numerous, tail conoid to long, filiform, similar in sexes.

Locality: Salcette Quepem Sanguem

Latitude: $15.3603 \mathrm{~N}, 15.2376 \mathrm{~N}, 15.1967 \mathrm{~N}$.

Longitude: 73.9234E, 74.2391E, 74.1195E.

Habitat: Near the roots of roadside weeds and bushy plants

Dimensions: Female: $L=2.58-3.05 \mathrm{~mm}, a=22-29$, $b=4.8-5.2, c=4.8-6.2, V=43-45$, odontostyle $=32-33 \mu \mathrm{m}$.

Male: $L=1.91-2.25 \mathrm{~mm}, a=31-39, b=5.0, c=11-13$, odontostyle $=34-35, \quad$ spicules $=76 \mu \mathrm{m}$, supplement number $=23-25$.

Prodorylaimus obesus Ahmad \& Jairajpuri, 1982

Body length usually 1-3 mm, female body curved upon fixation, tapering towards extremities, cuticle with transverse striations, guiding ring 'double', tail elongateconoid and dorsally curved, caudal pores on either side.

Locality: Canacona Mormugao Salcette

Latitude: $15.01415 \mathrm{~N}, 15.414 \mathrm{~N}, 15.3603 \mathrm{~N}$

Longitude: $74.05711 \mathrm{E}, 73.81 \mathrm{E}, 73.9234 \mathrm{E}$.

Habitat: Near the roots of bushy plants and roadside weeds.

Dimensions: Female: $\mathrm{L}=1.64-1.80 \mathrm{~mm}, \mathrm{a}=25-30$, $b=4.5-5.2, \quad c=27-30, \quad V=50-56, \quad G 1=22-27, \quad G 2=20$
27, odontostyle $=23-25$, odontophore $=32-33$, esophagus $=346-365 \mu \mathrm{m}$, tail $=61-70 \mu \mathrm{m}, A B D=30-33 \mu \mathrm{m}$.

Mesodorylaimus mesonyctius (Kreis, 1930) Andrassy, 1959

Body $1-2 \mathrm{~mm}$ long, cuticle thick with transverse striations, lip region continuous and angular, odontophore rod-like, oesophagus muscular and enlarging gradually, abrupt near the middle, vulval lips strongly cuticularised, female tail elongate-conoid in anterior and dorsally curved in posterior.

Locality: Canacona Mormugao Salcette Quepem Sanguem

Latitude: $15.01415 \mathrm{~N}, 15.414 \mathrm{~N}, 15.3603 \mathrm{~N}, 15.2376 \mathrm{~N}$, $15.1967 \mathrm{~N}$.

Longitude: 74.05711E, 73.81E, 73.9234E, 74.2391E, 74.1195E.

Habitat: Near the roots of flowering plants in the gardens, paddy fields.

Dimensions: Female: $\mathrm{L}=1.02-1.12 \mathrm{~mm}, \mathrm{a}=31.5-$ 34.6, b=4.2-4.9, $c=30.2-37.3, c^{\prime}=1.2-1.6, V=52.0-55.6$, $\mathrm{G} 1=12.3-26.0, \quad \mathrm{G} 2=14.6-27.2$, odontostyle=10-12, odontophore $=12.5-17$, tail $=30.5-37.0$, prerectum $=51.5-$ 73 , rectum $=22-36$.

Male: $L=0.99-1.05 \mathrm{~mm}, a=28-33, b=4.1-4.5, c=47.6-$ 61.8, $\quad c^{\prime}=0.7-0.9, \quad V=51.9-55.1$, odontostyle=11-12, odontophore $=13.5-17.5$, tail length $=17-22$, prerectum $=76-115$, rectum $=27-34$, spicule $=37-44$, ventromedian supplements=9-11.

Thornenema baldum (Thorne, 1939) Andrassy, 1959

Female $0.3-3 \mathrm{~mm}$ long, lip region offset by depression, sclerotised labial framework, odontostyle cylindrical, oesophagus expanded basally, female reproductive system amphidelphic, tail elongate-conoid to long, filiform in females; obtusely conoid in males.

Locality: Quepem Sanguem Canacona

Latitude: $15.2376 \mathrm{~N}, 15.1967 \mathrm{~N}, 15.01415 \mathrm{~N}$.

Longitude: 74.2391E, 74.1195E, 74.05711E.

Habitat: Near the roots of casuarina plantation and forest area.

Dimensions: Female: $\mathrm{L}=1.0-1.2 \mathrm{~mm}, \mathrm{a}=26-31$, $b=4.8-5.2, c=8.2-9.7, c^{\prime}=5.6-6.5, V=33-35, G 1=11.1-$ $12.5, \mathrm{G} 2=12-18$.

Thornenema lissum (Thorne, 1939) Andrassy, 1959

Body length $0.3-2.0 \mathrm{~mm}$, lip region rounded continuous with body contour, labial framework sclerotised, basal part of oesophagus expanded, female reproductive system mono-opisthodelphic, female tail long to filiform, male tail obtusely conoid. 
Locality: Mormugao Quepem Salcette Latitude: $15.414 \mathrm{~N}, 15.2376 \mathrm{~N}, 15.3603 \mathrm{~N}$ Longitude: 73.81E, 74.2391E, 73.9234E.

Habitat: Near the roots of vegetable plantation, flowering plants.

Coomansinema dimorphicauda Ahmad \& Jairajpur 1989

Body curved upon fixation, cuticle thick with fine transverse striae, lip region amalgated and continuous with body, odontostyle massive and wide, odontophore rod-like, female system amphidelphic, vagina with strong distal sclerotisation, female tail short, convexconoid with rounded tip and slight projection, male tail short, conoid.

Locality: Quepem Salcette Canacona

Latitude: $15.2376 \mathrm{~N}, 15.3603 \mathrm{~N}, 15.01415 \mathrm{~N}$

Longitude: 74.2391E, 73.9234E, 74.05711E

Habitat: Near the roots of plants in scrub jungle, roadside weeds

Dimensions: Female: $\mathrm{L}=1.25 \mathrm{~mm}, \mathrm{a}=25, \mathrm{~b}=3.8, \mathrm{c}=43$, $\mathrm{V}=56, \mathrm{G} 2=13$, odontostyle $=22 \mu \mathrm{m}$, odontophore $=24 \mu \mathrm{m}$, esophagus $=328-330 \mu \mathrm{m}$, prerectum $=71 \mu \mathrm{m}$, rectum $=$ $36 \mu \mathrm{m}$, tail $=29 \mu \mathrm{m}, \mathrm{ABD}=27-30 \mu \mathrm{m}$.

Baqriella qaiseri Ahmad \& Jairajpuri, 1988

Body length $0.7 \mathrm{~mm}$, small, slender, outer margin of lips forms a flap over oral area, odontostyle attenuated, three cardiac glands present at the oesophago-intestinal junction, vagina sclerotised distally, tail elongate-conoid, dorsally bent.

Locality: Sanguem Salcette

Latitude: $15.1967 \mathrm{~N}, 15.3603 \mathrm{~N}$

Longitude: 74.1195E, 73.9234E

Habitat: Near the roots of the plants in forest area

Dimensions: Female: $\mathrm{L}=0.73-0.77 \mathrm{~mm}, \mathrm{a}=22-23$, $b=3.3-3.5, \quad c=18-20, \quad c^{\prime} 2.3-3.0, \quad V=49-52, \quad G 1=10$, $\mathrm{G} 2=10-11$, odotostyle $=12.0-12.5$, odontophore $=17-18$, esophagus $=213-233 \mu \mathrm{m}$, prerectum $=35 \mu \mathrm{m}$, rectum $=$ $19 \mu \mathrm{m}$, tail $=38-42 \mu \mathrm{m}, \mathrm{ABD}=14-16 \mu \mathrm{m}$.

Ecumenicus monohystera (De Man, 1880) Thorne, 1974

Small nematode, $1.0-1.2 \mathrm{~mm}$ long, lips distinct offset by a slight depression, guiding ring 'single', vulva a transverse slit, vagina extending posteriorly, female reproductive system mono-opisthodelphic, tail blunt and digitate.

Locality: Canacona Mormugao Salcette Quepem Sanguem

Latitude: $15.01415 \mathrm{~N}, 15.414 \mathrm{~N}, 15.3603 \mathrm{~N}, 15.2376 \mathrm{~N}$,
$15.1967 \mathrm{~N}$

Longitude: 74.05711E, 73.81E, 73.9234E, 74.2391E, 74.1195E

Habitat: Near the roots of paddy plants

Dimensions: Female: $\mathrm{L}=1.05-1.16 \mathrm{~mm}, \quad \mathrm{a}=35-$ 39, $b=4.8-5.1, \quad c=30-34, \quad V=35-36, \quad G 2=13-15$, odontostyle $=11-12$, odontophore $=16-17$, esophagus= $214-230 \mu \mathrm{m}$, prerectum $=35-51 \mu \mathrm{m}$, rectum $=20-23 \mu \mathrm{m}$, tail $=33-35 \mu \mathrm{m}, \mathrm{ABD}=20-23 \mu \mathrm{m}$.

\section{Labronema ferox Thorne, 1939}

Body robust, $1.0-3.5 \mathrm{~mm}$ long, cuticle finely striated, lip region offset by deep constriction, broad and thick, odontostyle with side lumen and aperture, odontophore rod-like, guiding ring sclerotised, oesophagus enlarging gradually, vulva longitudinal, tail hemispheroid.

Locality: Quepem Sanguem Canacona

Latitude: $15.2376 \mathrm{~N}, 15.1967 \mathrm{~N}, 15.01415 \mathrm{~N}$

Longitude: 74.2391E, 74.1195E, 74.05711E

Habitat: Near the roots of plants in the forest area and scrub jungle.

Eudorylaimus himalus Jairajpuri \& Ahmad, 1982

Body curved upon fixation more posteriorly, odontostyle attenuated, cardia short and rounded, cardiac disc present, tail short and conoid with 'saccate bodies on the ventral side, pair of caudal pores on either side.

Locality: Sanguem Canacona Salcette Quepem

Latitude: $15.1967 \mathrm{~N} 15.01415 \mathrm{~N} 15.3603 \mathrm{~N} 15.2376 \mathrm{~N}$

Longitude: 74.1195E 74.05711E 73.9234E 74.2391E

Habitat: Near the roots of teak and acacia trees

Dimensions: Female: $\mathrm{L}=1.20-1.31 \mathrm{~mm}, \mathrm{a}=21-31$, $b==3.6-3.9, c=53-57, c^{\prime}=0.9-1.0, V=51-55, G 1=13-14$, $\mathrm{G} 2=10-13$, odontostyle=21-23, odontophore $=34-39$, esophagus $=309-363 \mu \mathrm{m}$, prerectum $=54-67 \mu \mathrm{m}$, rectum $=$ 23-25 $\mu \mathrm{m}$, tail $=22-24 \mu \mathrm{m}, \mathrm{ABD}=24-26 \mu \mathrm{m}$.

\section{Discolaimus texanus Cobb, 1913}

Body 1.2-2.7 mm long, lip region discoid and widely expanded, odontostyle long narrow thick walled, guiding ring single, basal expanded part of oesophagus marked off from anterior slender part by abrupt expansion, cardia disc-like with a blunt conoid part female reproductive system amphidelphic, tail bluntly rounded with obtuse tip.

Locality: Salcette

Latitude: $15.3603 \mathrm{~N}$

Longitude: 73.9234E

Habitat: Near the roots of vegetable plants

Dimensions: Female: $\mathrm{L}=1.52-1.62 \mathrm{~mm}, \mathrm{a}=47.7-$ 
55.8, b=4.9-5.4, c=36.3-40.7, $\quad c^{\prime}=2.3-2.5, \quad V=43-48$, odontostyle $=10-12$, odontophore $=13-20$, stylet length= 24-32, esophagus length $=300-310$, tail length $=40-43$, prerectum $=11-14$, rectum $=16-24, \mathrm{G} 1=85-118, \mathrm{G} 2=78-$ 115.

Discolaimus laksi Khan \& Laha, 1982

Female body arcuate upon fixation, lip region distinctly set off from the body, odontophore slightly longer than the odontostyle, reproductive system amphidelphic, tail dorsally convex and conoid.

Locality: Salcette

Latitude: $15.3603 \mathrm{~N}$

Longitude: 73.9234E

Habitat: Near the roots of banana plantation

Dimensions: Female: $\mathrm{L}=0.6-1.08 \mathrm{~mm}, \mathrm{a}=34-35$, $b=3.1-4.1, c=38-47, V=48-49$.

Enchodelus (Parenchodelus) constrictus Jairajpuri \& Loof, 1968

Body curved ventrally upon fixation, more towards the posterior half, lip region slightly set off, ovaries reflexed, tail short-conoid and ventrally arcuate.

Locality: Quepem Sanguem

Latitude: $15.2376 \mathrm{~N}, 15.1967 \mathrm{~N}$

Longitude: $74.2391 \mathrm{E}, 74.1195 \mathrm{E}$

Habitat: Near the roots of roadside weeds and forest plants

Dimensions: Female: $L=1.40 \mathrm{~mm}, a=24, b=5.2, c=36$, $c^{\prime}=1.5, V=51, \mathrm{G} 1=26, \mathrm{G} 2=30$.

Male: $L=1.19 \mathrm{~mm}, a=23, b=5.1, c=32, c^{\prime}=1.6, T=51$, Spicule $=46-48 \mu \mathrm{m}$.

Enchodelus (Parenchodelus) longidens Jairajpuri \& Loof, 1968

Body ventrally curved to C-shaped, odontophore linear, without flanges, reproductive amphidelphic, tail long, conoid provided with two caudal pores on either side.

Locality: Mormugao

Latitude: $15.414 \mathrm{~N}$

Longitude: $73.81 \mathrm{E}$

Habitat: Near the roots of bushy plants

Dimensions: Female: $L=1.58 \mathrm{~mm}, \mathrm{a}=35, \mathrm{~b}=4.5-5.3$, $c=32-35, c^{\prime} 1.5-1.7, V=48-51, G 1=18, G 2=20$

Male: $\mathrm{L}=1.64 \mathrm{~mm}, \mathrm{a}=33, \mathrm{~b}=5.2, \mathrm{c}=29, \mathrm{c}^{\prime}=1.8$, Tail $=65$.

Oriverutus labiatus Ahmad \& Jairajpuri, 1987

Body curved ventrally upon fixation, tapering towards the extremities, odontostyle attenuated, tail elongate conoid and dorsally bent.
Locality: Mormugao Salcette

Latitude: $15.414 \mathrm{~N} 15.3603 \mathrm{~N}$

Longitude: 73.81E 73.9234E

Habitat: Near the roots of bushy plants and roadside weeds

Dimensions: Female: $\mathrm{L}=0.88-1.1 \mathrm{~mm}, \mathrm{a}=31-37$, $b=3.4-3.6, c=21-24, c^{\prime}=2.2-2.6, T=52-60, G 2=13-16$, odontostyle $=15-16 \mu \mathrm{m}$, odontophore $=19-20 \mu \mathrm{m}$, esophagus $=253-278 \mu \mathrm{m}$, prerectum $=39-44 \mu \mathrm{m}$, rectum $=24-27 \mu \mathrm{m}$, tail $=41-45 \mu \mathrm{m}, \mathrm{ABD}=17 \mu \mathrm{m}$.

Oriverutus paragus Ahmad \& Jairajpuri, 1987

Body slightly curved ventrally upon fixation, odontostyle attenuated, well developed lateral guiding pieces, tail elongate conoid and ventrally curved with two caudal pores on either side.

Locality: Sanguem Canacona

Latitude: $15.1967 \mathrm{~N}, 15.01415 \mathrm{~N}$

Longitude: 74.1195E, 74.05711E

Habitat: Near the roots of forest trees

Dimensions: Female: $\mathrm{L}=0.98-1.22 \mathrm{~mm}, \mathrm{a}=26-28$, $b=3.1-3.8, c=20-22, c^{\prime}=2.2-2.6, V=48-52, G 1=17-19$, $\mathrm{G} 2=16-21$, odontostyle $=17-18 \mu \mathrm{m}$, odontophore $=22-24$ $\mu \mathrm{m}$, esophagus $=309-311$, prerectum $=55-666 \mu \mathrm{m}$, rectum $=21-22 \mu \mathrm{m}$, tail $=50-56 \mu \mathrm{m}, \mathrm{ABD}=21-23 \mu \mathrm{m}$.

Aporcelaimellus obscurus (Thorne \& Swanger, 1936) Heyns, 1965

Medium-sized nematode with robust body, cuticle finely striated, lip region set off with deep constriction, oral aperture hexagonal, female reproductive system amphidelphic, tail short and conoid.

Locality: Mormugao Salcette

Latitude: $15.414 \mathrm{~N}, 15.3603 \mathrm{~N}$

Habitat: Near the roots of paddy plants

Dimensions: Female: $\mathrm{L}=2.64-2.88 \mathrm{~mm}, \mathrm{a}=28-35$, $b=3.2-4.2, c=61-67, V=51-57, G 1=11-13, G 2=11-17$, odontostyle $=24-26 \mu \mathrm{m}$, odontophore $=42-46 \mu \mathrm{m}$, esophagus $=657-684 \mu \mathrm{m}$, prerectum $=120-139 \mu \mathrm{m}$, rectum $=57-60 \mu \mathrm{m}$, tail $=42 \mu \mathrm{m}, \mathrm{ABD}=48-53 \mu \mathrm{m}$.

Aporcelaimellus baqrii Ahmad \& Jairajpuri, 1982

Body ventrally curved upon fixation, reproductive system amphidelphic, female tail hemispheroid, irregularly spaced ventromedian supplements, tail short and conoid, pair of caudal pores on either side.

Locality: Mormugao Salcette

Latitude: $15.414 \mathrm{~N} 15.3603 \mathrm{~N}$

Longitude: 73.81E 73.9234E

Habitat: Near the roots of bushy plants and roadside weeds. 
Dimensions: Female: $\mathrm{L}=1.82-2.06 \mathrm{~mm}, \mathrm{a}=28-32$, $b=3.6-3.8, \quad c=63-67, \quad V=53-57, \quad G 1=7-8, \quad G 2=8-9$, odontostyle $=42-56$, odontophore $=38-41$, esophagus $=$ 507-544 $\mu \mathrm{m}$, prerectum $=45-67 \mu \mathrm{m}$, rectum $=41-45 \mu \mathrm{m}$, tail=27-30 $\mu \mathrm{m}, \mathrm{ABD}=32-36 \mu \mathrm{m}$.

Male: $L=1.86 \mathrm{~mm}, \quad a=29, \quad b=3.5 \quad c=58, \quad T=45$, odontostyle $=24$, odontophore $=39$, esophagus $=540 \mu \mathrm{m}$, spicules $=63 \mu \mathrm{m}$, lateral guiding piece $=16 \mu \mathrm{m}$, ventromedian supplements $=12$, prerectum $=65 \mu \mathrm{m}$, tail $=32 \mu \mathrm{m}, \mathrm{ABD}=38 \mu \mathrm{m}$.

Aporcelaimus regius (De Man, 1876) Thorne \& Swanger, 1936

Large-sized nematode, cuticle with criss-cross lines, lips region off set by deep constriction, odontostyle wide, aperture occupying more than one-half length, cardiac disc present, tail short, conoid to bluntly rounded.

Locality: Canacona

Latitude $15.01415 \mathrm{~N}$

Longitude: $74.05711 \mathrm{E}$

Habitat: In the soil around the roots of vegetable plants.

Longidorus brevicaudatus (Schuurmans Stekhoven, 1951) Khan, 1987

Female body tapering towards the extremities, lip region knob-like, reproductive system amphidelphic, tail cylindrical with rounded terminus.

Locality: Sanguem

Latitude: $15.1967 \mathrm{~N}$

Longitude: 74.1195E

Habitat: In the soil near the roots of the forest plants

Dimensions: Female: L=3.6-3.9 mm, $a=126-137$, $b=11.4-14.8, c=87.6-107.5, V=49-53$.

Longidorus elongatus (De Man, 1876) Thorne \& Swanger, 1936

Body long and elongated, lips amalgated, odontostyle long and attenuated, odontophore without basal flanges, female reproductive system amphidelphic, tail short, conoid and bluntly rounded.

Locality: Sanguem

Latitude: $15.1967 \mathrm{~N}$

Longitude: 74.1195E

Habitat: In the soil near the roots of the plants of forest area

Dimensions: Female: $\mathrm{L}=5.2-6.1 \mathrm{~mm}, \quad \mathrm{a}=78.6$ 106.6, $\quad b=11.5-13.5, \quad c=87.0-121.5, \quad V=45.6-53.1$, odontostyle=79-91 $\mu \mathrm{m}$, odontophore=57.5 $-66.5 \mu \mathrm{m}$, tail length $=44.5-59.0$.
Xiphinema insigne Loos, 1949

Female body long and slender with rounded anterior and elongate-conoid posterior extremity, odontophore nearly $2 / 3^{\text {rd }}$ of the odontostyle, tail narrow, female elongate-conoid and filiform, male tail conoid ending in a digitate terminus with two papillae on either side

Locality: Mormugao Salcette

Latitude: $15.414 \mathrm{~N}, 15.3603 \mathrm{~N}$

Longitude: $73.81 \mathrm{E}, 73.9234 \mathrm{E}$

Habitat: In the soil near the roots of vegetable plants and sapota plants

Dimensions: Female: $\mathrm{L}=1.8-2.6 \mathrm{~mm}, \mathrm{a}=51-71, \mathrm{~b}=5.3-$ 8.0, $c=15-35, c^{\prime}=3.1-8.6, V=28-36$, odontostyle $=80-111$ $\mu \mathrm{m}$, odontophore $=55-64 \mu \mathrm{m}$, guiding ring $=80-110 \mu \mathrm{m}$.

Male: $L=2.1-2.3 \mathrm{~mm}, a=52-65, b=5.4-6.4, c=47-54$, $c^{\prime}=1.4-1.7$, odontostyle $=93-104 \mu \mathrm{m}$, odontophore $=51-$ $61 \mu \mathrm{m}$, guiding ring $=87-98 \mu \mathrm{m}$, spicule $=53-58 \mu \mathrm{m}$.

Xiphinema americanum Cobb, 1913

Body long and slender, lip region continuous, odontostyle long and attenuated, odontophore with well developed basal flanges, female reproductive system amphidelphic, tail short and conoid.

Locality: Sanguem Canacona Quepem

Latitude $15.1967 \mathrm{~N}, 15.01415 \mathrm{~N}, 15.2376 \mathrm{~N}$

Longitude: 74.1195E, 74.05711E, 74.2391E

Habitat: In the soil near the roots of teak trees

Dimensions: Female: $\mathrm{L}=0.8-1.6 \mathrm{~mm}, \mathrm{a}=37-40$, $b=5.2-6.7, c=56-57, V=55-56$

\section{Axonchium (Axonchium) amplicolle Cobb, 1920}

Body length 1.1-3.0mm, straight to ventrally arcuate body upon fixation, lip region offset with inner section prominently demarcated, odontostyle fusiform, female body broadly rounded, male tail hemispheroid, conoid with broadly rounded tip.

Locality: Quepem Salcette Canacona

Latitude: $15.2376 \mathrm{~N}, 15.3603 \mathrm{~N}, 15.01415 \mathrm{~N}$

Longitude: 74.2391E, 73.9234E, 74.05711E

Habitat: In the soil near the roots of roadside plants and bushy plants.

Axonchium (Epaxonchium) vulvulatum Nair \& Coomans, 1974

Body usually 1.6-3.5 mm, ventrally curved upon fixation, conoid lips, odontostyle fusiform, constriction between the two parts of the oesophagus, vaginal wall internally sclerotised, female tail hemispherical, spaced ventromedian supplements, male tail convex-conoid.

Locality: Quepem Salcette Canacona Sanguem

Latitude: $15.2376 \mathrm{~N}, 15.3603 \mathrm{~N}, 15.01415 \mathrm{~N}, 15.1967 \mathrm{~N}$ 
Longitude: 74.2391E, 73.9234E, 74.05711E, 74.1195E.

Habitat: Near the roots of vegetable plants, flower gardens, bushy plants

\section{Order Mononchida}

Mononchus aquaticus Coetzee, 1968

Lip region 18-22 $\mu \mathrm{m}$ wide, 6-8 $\mu \mathrm{m}$, buccal cavity28-37 $\mu \mathrm{m}$ long and 13-16 $\mu \mathrm{m}$ wide, dorsal tooth of medium size, situated in the anterior half of the buccal cavity, subventral walls without any denticles but with an indentation opposite dorsal tooth apex, reproductive system amphidelphic, presence of an sphincter at oviduct-uterus junction, tail elongate conoid and clavate at tip, caudal glands well developed and terminal opening present.

Locality: Quepem Salcette

Latitude: $15.2376 \mathrm{~N}, 15.3603 \mathrm{~N}$

Longitude: 74.2391E, 73.9234E

Habitat: In the soil around the roots of banana plants

Dimensions: Female: $\mathrm{L}=1.4-1.7 \mathrm{~mm}, \mathrm{a}=33-36$, $b=3.8-4.3, c=8.5-10.0, V=52-54$

\section{Mononchus tunbridgensis Bastian, 1865}

Lip region 10-15 $\mu \mathrm{m}$ wide, 4-5 $\mu \mathrm{m}$ high, buccal cavity 18-20 $\mu \mathrm{m}$ long and $6-7 \mu \mathrm{m}$ wide, dorsal tooth of medium size, situated in anterior half of buccal cavity, subventral walls without any denticle but with an indentation opposite dorsal tooth apex, reproductive system amphidelphic, sphincter present at oviductuterus junction, tail first conoid then sharply cylindroid, caudal glands and terminal opening present.

Locality: Sanguem Salcette

Latitude: $15.1967 \mathrm{~N}, 15.3603 \mathrm{~N}$

Longitude: 74.1195E, 73.9234E

Habitat: In the soil around the roots of casuarina and acacia trees

Dimensions: Female: $\mathrm{L}=1.03-1.15 \mathrm{~mm}, \mathrm{a}=26-30$, $b=3.7-4.1, c=9-12, V=53-57$

Iotonchus trichurus (Cobb, 1917) Altherr, 1958

Lip region 24-30 $\mu \mathrm{m}$ wide, 7-12 $\mu \mathrm{m}$ high, amphidial aperture 4-6 $\mu \mathrm{m}$ wide, buccal cavity $26-33 \mu \mathrm{m}$ long and 16-19 $\mu \mathrm{m}$, dorsal tooth small and basal, its apex 5-8 $\mu \mathrm{m}$ from the base of buccal cavity, reproductive system mono-prodelphic, sphincter not present at oviductuterus junction, tail long, whip-like, caudal glands present and terminal opening present.

Locality: Sanguem Salcette

Latitude: $15.1967 \mathrm{~N}, 15.3603 \mathrm{~N}$

Longitude: 74.1195E, 73.9234E
Habitat: In the soil around the roots of casuarina and acacia trees

Dimensions: Female: $L=1.5-1.9 \mathrm{~mm}, \mathrm{a}=30-45, \mathrm{~b}=3.6-$ $4.8, c=3-5, V=54-65$.

lotonchus indicus Jairajpuri, 1969

Female lip region 37-44 $\mu \mathrm{m}$ wide, 14-16 $\mu \mathrm{m}$ high, amphidial aperture 5-7 $\mu \mathrm{m}$ wide, located at 12-16 $\mu \mathrm{m}$ from anterior end of body and 35-42 $\mu \mathrm{m}$ from base of buccal cavity, buccal cavity 40-47 $\mu \mathrm{m}$ long, and 28-32 $\mu \mathrm{m}$ wide, dorsal tooth small and basal, excretory system not observed, reproductive system amphidelphic, sphincter not present at oviduct-uterus junction, tail elongate, conoid and tapering regularly, caudal glands poorly developed and opening subterminal dorsally.

Locality: Quepem Salcette

Latitude: $15.2376 \mathrm{~N}, 15.3603 \mathrm{~N}$

Longitude: $74.2391 \mathrm{E}, 73.9234 \mathrm{E}$

Habitat: In the soil around the roots of banana plants and mango and casuarina trees

Dimensions: Female: L=1.55-1.98 mm, $a=21-32$, $b=4.1-4.9, c=5-8, V=57-65$.

Parahadronchus shakili (Jairajpuri, 1969) Mulvey, 1978

Female length 2.22-3.30 mm, male length 2.04$2.80 \mathrm{~mm}$, lip region 42-60 $\mu \mathrm{m}$ wide, 16-23 $\mu \mathrm{m}$ high, amphidial aperture 5-6 $\mu \mathrm{m}$, buccal cavity 46-65 $\mu \mathrm{m}$ long, 30-40 $\mu \mathrm{m}$ wide, dorsal tooth large and suprebasal, subventral walls bears 3-6 teeth, excretory system not observed, reproductive system amphidelphic, well developed sphincter present at oviduct-uterus junction, tail elongate-conoid, tapering sharply, caudal glands well developed opening terminal.

Locality Salcette Canacona

Latitude: $15.3603 \mathrm{~N}, 15.01415 \mathrm{~N}$

Longitude: 73.9234E, 74.05711E

Habitat: In the soil around the roots of banana plants casuarina trees

Dimensions: Female: $\mathrm{L}=2.23-3.30 \mathrm{~mm}, \mathrm{a}=32-42$, $b=4.2-4.9, c=7-11, V=60-70$.

Male: $L=2.05-2.81 \mathrm{~mm}, \mathrm{a}=28-42, \mathrm{~b}=3.5-4.67 \mathrm{c}=9-$ $11, T=42-51$.

Parahadronchus andamanicus (Jairajpuri, 1969) Mulvey, 1978

Female length 2.24-3.22 mm, male length 2.21-2.78 $\mathrm{mm}$, female lip region 46-65 $\mu \mathrm{m}$ wide and 18-24 $\mu \mathrm{m}$ high, buccal cavity 58-77 $\mu \mathrm{m}$ long, 35-44 $\mu \mathrm{m}$ wide, dorsal tooth large and suprabasal, its apex 26-35 $\mu \mathrm{m}$ or $43-45 \%$ of the length of buccal cavity from base, 
each subventral wall bearing denticulate ridge having 4-8 small denticles, excretory system not observed, reproductive system mono-prodelphic, posterior uterine sac present, well developed sphincter present at oviductuterus junction, tail long filiform, caudal glands present, opening terminal.

Locality: Salcette Canacona

Latitude: $15.3603 \mathrm{~N} 15.01415 \mathrm{~N}$

Longitude: 73.9234E 74.05711E

Habitat: In the soil around the roots of arecanut and banana plants

Dimensions: Female: $\mathrm{L}=2.25-3.23 \mathrm{~mm}, \mathrm{a}=30-41$, $b=3.8-4.45 c=5-13, V=64-75$.

Male: $L=2.21-2.78 \mathrm{~mm}, a=32-42, b=4.0-4.4, c=6-8$, $\mathrm{T}=\mathbf{2 5}-\mathbf{4 5}$.

\section{Order Tylenchida}

Tylenchus filiformis Bütschli, 1873

Body cylindrical from medium to vulva and tapering uniformly to the acute terminus, tail twice as long as vulva-anus distance, stylet with small rounded knobs, median bulb ovate, nerve ring near middle of slender isthmus, basal bulb elongate and pyriform.

Locality: Sanguem Salcette

Latitude: $15.1967 \mathrm{~N}, 15.3603 \mathrm{~N}$

Longitude: 74.1195E, 73.9234E

Habitat: In the soil around the roots of vegetable plants

Dimensions: Female: $\mathrm{L}=0.46 \mathrm{~mm}, \mathrm{a}=26, \mathrm{~b}=5.5, \mathrm{c}=3.2$ $\mathrm{V}=58$, stylet $=11 \mu \mathrm{m}$.

Tylenchus indicus Khan et al., 1969

Female length $0.40-0.50 \mathrm{~mm}$, head slightly off set, cuticle annulated, spear well developed and knobbed, median bulb oval, bursa moderately developed and tail relatively long and slender.

Locality: Quepem Canacona

Latitude: $15.2376 \mathrm{~N}, 15.01415 \mathrm{~N}$

Longitude: 74.2391E, 74.05711E

Habitat: In the soil around the roots of vegetable plants and paddy fields

Dimensions: Female: $\mathrm{L}=0.40-0.50 \mathrm{~mm}, \mathrm{a}=20-26$, $c=5.0-6.0, c^{\prime} 6-19, V=65-68$, spear $=12 \mu \mathrm{m}$, annules in pharyngeal region $=46$, tail $=80-83 \mu \mathrm{m}$.

Ottolenchus parvus (Siddiqi, 1963) Siddiqi, 1979

Female length 0.38-0.54 mm, cuticular annule, head smooth, 37-45 annules drawn from anterior body end to excretory pore, vagina about half body width long, tail tip pointed or finely rounded.

Locality: Quepem Canacona
Latitude: $15.2376 \mathrm{~N}, 15.01415 \mathrm{~N}$

Longitude: 74.2391E, 74.05711E

Habitat: In the soil around the roots of vegetable plants

Dimensions: Female: $L=0.39-0.54 \mathrm{~mm}, a=27-33$, $b=5.4-7.2, c=3.9-4.7, V=58-66$, stylet $=7-8 \mu \mathrm{m}$.

Male: $\mathrm{L}=0.45 \mathrm{~mm}, \mathrm{a}=31, \mathrm{~b}=6, \mathrm{c}=4$, spicules $=14 \mu \mathrm{m}$.

Psilenchus minor Siddiqi, 1963

Female length $0.85 \mathrm{~mm}$, male length $0.89 \mathrm{~mm}$, lip region rounded, smooth and continuous with body contour, lateral fields marked by four incisures, tail elongate-filiform, regularly tapering to a bluntly rounded tip, bursa adanal, crenate arising near head of spicules and extending up to phasmid.

Locality: Mormugao

Latitude: $15.414 \mathrm{~N}$

Longitude: $73.81 \mathrm{E}$

Habitat: In the soil around the roots of bushy plants

Dimensions: Female: $L=0.85 \mathrm{~mm}, a=45, b=5.7, c=5.9$, $c^{\prime}=11, V=47.7$, stylet $=13 \mu \mathrm{m}$.

Male: $L=0.89 \mathrm{~mm}, a=49, b=6.4, c=6.3$, spicule $=28 \mu \mathrm{m}$, gubernaculum $=6 \mu \mathrm{m}$.

Tylenchorhynchus dubius (Bütschli, 1873) Filipjev, 1936

Female $0.6-1.1 \mathrm{~mm}$ long male $0.6-0.9 \mathrm{~mm}$ long, head offset, hemispherical with 7 annules, body cylindrical tapering only slightly at either end, spear $19 \mu$ with fairly well developed, rounded with basal knobs, oesophgealintestinal valve hemispherical but inconspicuous, female tail cylindrical with bluntly rounded tip which is annulated, bursa beginning anterior to spicules and surrounding tail tip, gubernaculum slightly curved proximally.

Locality: Salcette Quepem

Latitude: $15.3603 \mathrm{~N}, 15.2376 \mathrm{~N}$

Longitude: 73.9234E, 74.2391E

Habitat: In the soil around the roots of teak trees and acacia trees

Dimensions: Female: $L=0.6-1.0 \mathrm{~mm}, \mathrm{a}=27-30, \mathrm{~b}=5-7$, $c=13-15, V=50-55$, stylet $=15-18 \mu \mathrm{m}$, lip annules=3, number of tail annules $=23$.

Male: $L=0.5-0.9 \mathrm{~mm}, a=26-33, b=4.7-6.9, c=10-$ 15 stylet $=15-18 \mu \mathrm{m}$, spicule=20, gubernaculum $=12$.

Hoplolaimus indicus Sher, 1963

Female 1.02-1.40 mm long and male 0.94-1.30 mm, cuticle coarsely annulated, lateral fields marked by single incisures or 2-3 incomplete broken incisures, lip region hemispherical, marked by 3-4 annules, basal annules 
with 6-12 longitudinal striations, cephalic framework strongly sclerotized, stylet robust, basal knobs tulip shaped, tail rounded with 8-13 annules, spicules arcuate and cephalated, bursa terminal.

Locality: Canacona Sanguem

Latitude: $15.01415 \mathrm{~N}, 15.1967 \mathrm{~N}$

Longitude: 74.05711E, 74.1195E

Habitat: In the soil near the roots of casuarina trees and in the forest area

Dimensions: Female: $\mathrm{L}=1.02-1.40 \mathrm{~mm}, \mathrm{a}=22-36$, $b=8.5-9.2, b^{\prime}=7.0-8.2, c=45-74, c^{\prime}=0.67-0.69, V=50-59$, stylet $=30-34 \mu \mathrm{m}$.

Male: $\mathrm{L}=0.94-1.30 \mathrm{~mm}, \mathrm{a}=26-36, \mathrm{~b}=8.9-12.1, \mathrm{~b}^{\prime}=6.2$ 9.1, $c=31-28$, spicule $=37-48 \mu \mathrm{m}$, gubernaculum $=12$ $20 \mu \mathrm{m}$.

Hoplolaimus seinhorsti Luc, 1958

Female length 1.1-1.6 mm, body cylindrical and cuticle coarsely annulated, head markedly offset,head skeleton powerfully built, female tail short, bluntly rounded with 10-15 annules going right round its tip, male tail surrounded by large bursa with a terminal unstriated lobe.

Locality: Quepem Canacoa

Latitude: $15.2376 \mathrm{~N}, 15.01415 \mathrm{~N}$

Longitude: $74.2391 \mathrm{E}, 74.05711 \mathrm{E}$

Habitat: In the soil around the roots of vegetable plants

Dimensions: Female: $\mathrm{L}=1.1-1.6 \mathrm{~mm}$, incisures $=1$, pharyngeal gland nuclei $=6$, stylet $=40-49$, labial annules $=4$, longitudinal striae on basal lip annule $=8-12$, tail annule $=10-15$

\section{Helicotylenchus indicus, Siddiqi, 1963}

Female length 0.45-0.54 mm, Body spirally curved, lip region conoid-rounded with 4-5 indistinct annules, lateral field $1 / 7^{\text {th }}$ of body width, marked with four incisures, continuous till the tip of the tail, tail hemispherical.

Locality: Quepem Salcette

Latitude: $15.2376 \mathrm{~N}, 15.3603 \mathrm{~N}$

Longitude: 74.2391E, 73.9234E

Habitat: In the soil around the roots of teak and casuarina trees

Dimensions: Female: $\mathrm{L}=0.45-0.54 \mathrm{~mm}, \mathrm{a}=23-25$, $b=4.5-5.8, b^{\prime}=3.5-5.2, c=24-29, c^{\prime}=1.2-1.6, \quad V=59-65$, stylet $=24-26 \mu \mathrm{m}$.

Criconemella xenoplax (Raski, 1952) Luc \& Raski, 1981

Female length $0.40-0.62 \mathrm{~mm}$, annules retrose (200 or fewer) with smooth or slightly rough posterior margins, especially towards the tail, head broad, first annule entire or emarginated laterally, lip region conspicuous, elevated, tail broadly rounded to more conoid, terminus generally a simple rounded or lobed button.

Locality: Salcette Quepem

Latitude: $15.3603 \mathrm{~N}, 15.2376 \mathrm{~N}$

Longitude: 73.9234E, 74.2391E

Habitat: In the soil near the roots of teak and casuarina trees

Dimensions: Female: $L=0.40-0.62 \mathrm{~mm}, \mathrm{a}=8-14, \mathrm{~b}=3-$ 5, c=23-56, V=90-95, stylet $=71-86 \mu \mathrm{m}$.

\section{Order Alaimida}

Alaimus primitivus de Man, 1880

Female body length 1.0-1.2 mm, body tapering evenly at both ends, lip region rounded, amphidial aperture minute, oesophagus in two parts, anterior narrow tubular and posterior expanding to form an elongated basal swelling, vulva a transverse slit, female reproductive system mono-opisthodelphic, tail elongate conoid and tapering to pointed terminus.

Locality: Salcette Canacona

Latitude: $15.3603 \mathrm{~N}, 15.01415 \mathrm{~N}$

Longitude: 73.9234E, 74.05711E

Habitat: From the soil around the roots of casuarina trees

Dimensions: Female: $\mathrm{L}=0.8-1.2 \mathrm{~mm}, \mathrm{a}=42-58$, $b=3.8-4.4, c=7.7-8.3, V=39-55$.

Male: $\mathrm{L}=0.76-0.94 \mathrm{~mm}, \mathrm{a}=55-75, \mathrm{~b}=4.5-4.8, \mathrm{c}=9-10$.

Alaimus hamulus Siddiqi \& Husain, 1967

Female body length $0.95-1.20 \mathrm{~mm}$, female body ventrally arcuate more posteriorly, cuticle with fine striae, lip region round continuous with body contour, nerve ring a little posterior to middle of neck, reproductive system mono-opisthodelphic, tail ventrally hooked tapering ends abruptly to a sharp point.

Locality: Quepem Sanguem

Latitude: $15.2376 \mathrm{~N}, 15.1967 \mathrm{~N}$

Longitude: 74.2391E, 74.1195E

Habitat: In the soil around the roots of sapota trees and acacia trees

Dimensions: Female: $L=0.95-1.20 \mathrm{~mm}, a=54-64$, $b=4.3-4.89 \mathrm{c}=11-15, \mathrm{~V}=42-46$.

Male: $L=1.0-1.2 \mathrm{~mm}, a=58-62, b=4.3-4.6, c=10-11$, $\mathrm{T}=40-48$.

Amphidelus novus Baqri \& Jairajpuri, 1968

Female body length $1.52-1.62 \mathrm{~mm}$, body slender tapering gradually anterior to slender part of pharynx, 
cuticle smooth, lip region narrow, slightly marked off from the body, amphid narrow and elongate, reproductive system amphidelphic, tail long and filiform, terminus finely rounded.

Locality: Quepem

Latitude: $15.2376 \mathrm{~N}$

Longitude: $74.2391 \mathrm{E}$

Habitat: From the soil around the roots of acacia trees

Dimensions: Female: $\mathrm{L}=1.52-1.62 \mathrm{~mm}, \mathrm{a}=59-68$, $b=5.7-6.1, c=6-9, V=32-33$.

Male: $\mathrm{L}=1.45-1.7 \mathrm{~mm}, \mathrm{a}=58-59, \mathrm{~b}=5.5-5.6, \mathrm{c}=8-9$.

\section{Order Rhabditida}

Caenorhabditis elegans (Maupas, 1899) Dougherty, 1953

Female body length $0.70-1.1 \mathrm{~mm}$, male body length $0.53-0.65 \mathrm{~mm}$, body tapering at extremities, lips bearing circlets of six, pharyngeal collar surrounds about half the stoma, oesophagus without a median bulb, basal bulb with valve, nerve ring near middle of isthmus, female tail conical, long

Locality: Canacona Sanguem

Latitude: $15.01415 \mathrm{~N}, 15.1967 \mathrm{~N}$

Longitude: 74.05711E, 74.1195E

Habitat: From the soil around the roots of casuarina trees

Dimensions: Female: $L=0.70-1.1 \mathrm{~mm}, a=19-21, b=6-$ $8, c=8-10, V=52-55$

Male: $\mathrm{L}=0.53-0.65 \mathrm{~mm}, \mathrm{a}=16-18, \mathrm{~b}=4.2-4.8, \mathrm{c}=22-$ 25.

\section{Cephalobus persegnis Bastian, 1865}

Female body length $0.50-.62 \mathrm{~mm}$, body spindle shaped more attenuated anteriorly, cuticle striated, lips low and rounded, obscurely duplex and median lip asymmetrical, oesophagus cylindrical and tapers to the isthmus from which it is separated only by a break in the musculature, female gonad single and prodelphic, female tail uniformly conoid to blunt terminus.

Locality: Salcette

Latitude: $15.3603 \mathrm{~N}$

Longitude: 73.9234E

Habitat: From the soil around the casuarina trees and vegetable plants

Dimensions: Female: $\mathrm{L}=0.50-0.62 \mathrm{~mm}, \mathrm{a}=21-23$, $b=3.9-4.9, c=13-15, V=68-69$.

Acrobeles timmi Chaturvedi \& Khera, 1979

Female length $0.4-0.52 \mathrm{~mm}$, body robust, tapering towards the extremities, cuticle thick and coarsely striated, cephalic probolae reaching half way to labials, acute forwardly pointing and lightly fringed, head off set, vulva post equatorial, ovary single, anterior and reflexed, tail conoid.

Locality: Canacona

Latitude $15.01415 \mathrm{~N}$

Longitude: $74.05711 \mathrm{E}$

Habitat: From the soil around the roots casuarina trees and banana plants

Dimensions: Female: $\mathrm{L}=0.4-0.52 \mathrm{~mm}, \mathrm{a}=16.8-18.7$, $b=3.6-3.8, c=10.2-11.7, V=60-61$.

\section{Panagrolaimus fuchsi Ruhm, 1956}

Female length $0.59-0.64 \mathrm{~mm}, 3$ or 6 lips amalgamated, cheilstom wider than long, rhabdions absent, prorhabdions longer than mesorhabdions, both swollen at the base, mesostom funnel shaped, female tail narrow conical with an offset tip.

Locality: Mormugao

Latitude: $15.414 \mathrm{~N}$

Longitude: $73.81 \mathrm{E}$

Habitat: From the soil around the roots of flower garden plants and bushy plants

Dimensions: Female: L=0.59-0.64 mm, $a=18-19$, $b=4.4-5.8, c=5.8-8.4, V=54-59$.

Male: $\mathrm{L}=0.59-0.64 \mathrm{~mm}, \mathrm{a}=23, \mathrm{~b}=5.4-5.8, \mathrm{c}=7.3-7.4$.

\section{Order Enoplida}

\section{Ironus longicaudatus De Man, 1884}

Female body length $1.4-1.6 \mathrm{~mm}$, body slender, tapering towards extremities, ventrally curved upon fixation, cuticle smooth, head set off, lips round, amphid cup-shaped, pharynx composed of sclerotized shields with three teeth at its base, one dorsal and two subventral, stoma tubular, oesophagus cylindrical, vulva pre-equatorial, ovary paired, opposed and reflexed, tail long and filiform.

Locality: Salcette Quepem

Latitude: $15.3603 \mathrm{~N}, 15.2376 \mathrm{~N}$

Longitude: 73.9234E, 74.2391E

Habitat: From the soil around the roots of mango and cashew trees

Dimensions: Female: $\mathrm{L}=1.4-1.6 \mathrm{~mm}, \mathrm{a}=50-55$, $b=5.3-6.1, c=4.1-4.9, V=42-44$.

Ironus ignavus Bastian, 1865

Female body length 1.40-1.54 mm, body slender, cuticle without striations head offset by shallow constriction, stoma long tubular with 3 anterior eversible hook-like tooth, vulva equatorial, gonads paired, opposed and reflexed, tail tapering to a fine point. 
Locality: Quepem Canacona Sanguem

Latitude: $15.2376 \mathrm{~N}, 15.01415 \mathrm{~N}, 15.1967 \mathrm{~N}$

Longitude: 74.2391E, 74.05711E, 74.1195E

Habitat: From the soil around the roots of banana plants and bamboo reeds

Dimensions: Female: $\mathrm{L}=1.40-1.54 \mathrm{~mm}, \mathrm{a}=40-50$, $b=5.2-5.9, c=4.7-5.3, V=45-49$.

\section{Order Araeolaimida}

\section{Plectus cirratus Bastian, 1865}

Female body length $0.90-0.93 \mathrm{~mm}$, body arcuate ventrally upon fixation, cuticle thick and finely striated, head slightly set off, oesophagus cylindrical with a valvular terminal bulb, ovaries two, opposed and reflexed, tail long tapering, terminus with spinneret.

Locality: Salcette

Latitude: $15.3603 \mathrm{~N}$

Longitude: 73.9234E

Habitat: From the soil of the paddy fields

Dimensions: Female: $\mathrm{L}=0.90-0.93 \mathrm{~mm}, \mathrm{a}=21-22$, $b=4.1-4.3, c=7.9-8.1, V=47-49$.

\section{References}

Ahmad, W. \& M.S. Jairajpuri (1987). Studies on the genus Oriverutus (Nematoda: Dorylaimida). Nematologica 33: 10-21.

Ahmad, W. \& M.S. Jairajpuri (1988). Baqriella qaiseri gen. n., sp. (Nematoda: Dorylaimida) from Mussoorie hills, India. Indian Journal of Nematology 18: 27-29.

Ahmad, W. \& M.S. Jairajpuri (1989). Coomansinema n. gen. (Nematoda: Dorylaimida) with the description of $C$. dimorphicauda n. sp. Nematologica 35: 142-146.

Andrassy, I. (1999). A census of genera and subgenera of free-living nematodes. Journal of nematode morphology and systematic 2 45-68.

Barker, K.R., R.S. Hussey, L.R. Krusberg, G.W. Bird, R.A. Dunn, H. Ferris, P.S. Ferris, D.W. Freckman, C.J. Gabriel, P.S. Grewal, A.E.
Macguidwin, D.L. Riddle, P. A. Roberts \& D.P. Schmitt (1994). Plant and soil nematodes: social impact and focus for the future. Journal of Nematology 6: 127-137.

Boag, B. \& G.W. Yeates (1998). Soil nematode biodiversity in terrestrial ecosystems. Biodiversity and Conservation 7: 617-630.

Bongers, T. (1990). The maturity index: an ecological measure of environmental disturbance based on nematode species composition. Oecologia 83: 14-19.

Choudhary, M., W. Ahmad \& M.S. Jairajpuri (2010). Alaimina FreeLiving Soil-Inhabiting Nematodes. Aligarh Muslim University Press, Aligarh, 156pp.

Cobb, N.A., (1919). Plant parasitic nematodes of India, An identification Manual. Ed. Wasim Ahmad. Litho offset Printers, Aligarh

de Maeseneer, J. \& J. d'Herde (1963). Methodes utilisees pour l'etude des anguillules libres du sol. Revue de l' Agriculture Bruxelles 16: 441-447.

Director, ZSI (2008) (Ed.). Fauna of Goa, State Fauna Series, 16. Zoological Survey of India, Kolkata, 531pp.

Esquivel, A. (2003). Nematode fauna of Costa Rican protected areas. Nematropica 33: 131-145.

Freckman, D.W. (1988). Bacterivorous nematodes and organic matter decomposition. Argiculture, Ecosystems and Environment 24: 195-217

Goafaoundation.org: NBSAPS Report: google.com - godfaoundation. org/completed-projects/biodiversity-in-goa/Checklist of Goa's Biodiversity <http://www.goacom.com/goafoundation/ biodiversity> Accessed on 20 January 2014.

Goodey, T. (1963). Soil and Freshwater Nematodes. Second Edition, revised and rewritten by J.B. Goodey, London: Methuen \& Co. Ltd.

Hugot, J.P., P. Baujard \& S. Morand (2001). Biodiversity in helminth nematodes as a field study: an overview. Nematology 3(3): 199208

Jairajpuri, M.S. \& W.U. Khan (1982). Predatory Nematodes (Mononchida). Associated Publishing Company, New Delhi, India, vi+131pp.

Jairajpuri, M.S. \& W. Ahmad (1992). Dorylaimida, Predaceous and Plant - Parasitic Nematodes. Oxford \& IBH Publ. Co.

Neher, D. (2001). Role of nematodes in soil health and their use as indicators. Journal of Nematology 33: 161-168.

Seinhorst, J.W. (1959). A rapid method for the transfer of nematodes from fixatives to anhydrous glycerin. Nematologica 4: 67-69.

Siddiqi, M.R. (2000). Tylenchida. Parasites of Plants and Insects. $2^{\text {nd }}$ Edition. CAB International Wallingford Oxon OX10 8DE. UK.

Min:

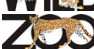

